\title{
Pathogen-imposed skewing of mouse chemokine and cytokine expression at the infected tissue site
}

\author{
Shoshana D. Katzman and Deborah J. Fowell \\ David H. Smith Center for Vaccine Biology and Immunology, Aab Institute of Biomedical Sciences, \\ Department of Microbiology and Immunology, University of Rochester, Rochester, New York, USA.
}

\begin{abstract}
Compartmentalization of immunity ensures tight regulation of T cell activation in the LN and precise effector $T$ cell delivery to inflamed sites. Herein we show that the tissue-specific accumulation of effector $T$ cells can be subverted by a pathogen at the infection site. Using the Leishmania major mouse model of dermal infection, we observed a restricted chemokine profile at the infection site, i.e., the expression of Th2 cell-attracting CCL7 but not of Th1 cell-attracting chemokines. Consistent with these chemokine expression data, recruitment of cytokine-producing $T$ cells to the infection site was also selective. Both IL-4- and IFN- $\gamma-$ producing effector $T$ cells homed to inflamed OVA/CFA-immunized dermis, but only IL-4-producing cells homed to $L$. major-infected dermis. The narrowing of the cytokine repertoire at the site of infection with $L$. major was driven, in part, by pathogen-induced CCL7. Inflammatory signals failed to disrupt the early restrictive L. major infection site, which suggests that $L$. major dominantly modifies the local milieu. We have highlighted an emerging principle in pathogen-host interactions: that the cytokine repertoire at the infection site and the LN draining the infection site can be different because of the ability of the pathogen to modify the chemokine profile at the infection site. Thus, pathogens may edit the LN cytokine repertoire through differential recruitment of cytokine-producing cells.
\end{abstract}

\section{Introduction}

The acquisition and delivery of $\mathrm{T}$ cell cytokine responses are anatomically compartmentalized. Immune responses following infection are often measured in the draining LN or peripheral blood, and it is unclear how well these responses are reflected in the effector response in the infected tissue. Studies in a number of infectious settings suggest that the tissue site may modulate cytokine production either by differential priming or recruitment/activation of effectors (1-3). Local immune modulation could be driven by the pathogen itself or by tissue- or organ-specific regulation of immunity $(4,5)$.

It is well established that dendritic cell activation and function is modulated through TLR ligation in response to pathogen encounter or tissue damage $(6,7)$. Such changes direct the subsequent selective priming of $\mathrm{T}$ cell effector function in the draining lymph node (dLN). During Th differentiation, Th1 and Th2 subsets also express a distinct array of chemokine receptors (8) and thus have the potential to be recruited to different inflammatory sites. In addition, $\mathrm{T}$ cells appear to acquire tissue site-specific homing receptors for guidance back to damaged or infected sites $(9,10)$. Therefore, both the effector function and homing potential of effector T cells may be predetermined during differentiation in the $\mathrm{LN}$ and directed by the type and location of danger signals.

In addition to pathogen-induced changes in the generation of the $\mathrm{T}$ cell effector repertoire, recent work suggests that the peripheral infected tissue site may have significant modulatory potential

Nonstandard abbreviations used: dLN, draining lymph node; ELISPOT, enzymelinked immunospot; IFA, incomplete Freund's adjuvant; pOVA, peptide OVA; SLA, soluble leishmania antigens.

Conflict of interest: The authors have declared that no conflict of interest exists. Citation for this article: J. Clin. Invest. 118:801-811 (2008). doi:10.1172/JCI33174. on the localization and/or activation of LN-derived antigen-specific effectors. Tissue-specific expression of STAT1 and STAT6 appears to be required for the trafficking of Th1 and Th2 effectors, respectively, to the lung $(11,12)$. In addition, there is compelling evidence that cytokine secretion also occurs in distinct locations (13-15), which raises the possibility that signals for activation and effector function may be site-specific. The distinct innate immune profile for individual tissues such as the lung and gut may contribute to differential recruitment or activation of $\mathrm{T}$ cell effector function $(1,2,16-18)$. Indeed, the dependency on CD40/CD40L interactions for differential effector function (Th1 versus Th2) was found to be tissue-specific during systemic infection with Listeria monocytogenes (1). Finally, the pathogen itself may shape the effector response in the infected tissue. Influenza infection of the lung was shown to inhibit the efficient recruitment of Th2 cells into the airways without altering the LN generation of Th2 cells (3). Similar mechanisms may explain the suppression of Th2 responses in the lung after mycobacterial infection (19). In schistosome granulomas, regulatory events constrain IFN- $\gamma$, but not IL-4, production within the granuloma (20). In addition, numerous viruses and Schistosoma mansoni have been shown to express chemokine decoys that limit the function of local proinflammatory chemokines $(21,22)$. Thus, site-specific alterations in immune function may significantly influence the outcome of infection. However, in many instances it has been difficult to separate the effects of pathogens on T cell priming in the dLN via APCs from the infection site from local effects of pathogens on $\mathrm{T}$ cell effector function at the site of the infection itself.

We focused on the local milieu at the dermal site of infection with the parasite Leishmania major (23). The effects of leishmania on immune activation at the infection site have primarily been focused on the initial activation of innate effectors. There is surprisingly lit- 
tle induction of macrophage activation following $L$. major infection compared with other intracellular pathogens $(24,25)$, which leads to the notion that initial L. major infection is a relatively "silent" affair. Far from being a passive event, Leishmania species have been shown to actively impede macrophage responses to other inflammatory stimuli, such as LPS $(26,27)$. How this modulation of the local environment early in infection influences subsequent recruitment/activation of antigen-specific T cells is not well understood. In this study we address the effects of $L$. major on the dermal tissue itself. Analysis of chemokine expression at the site of L. major infection revealed the presence of CCL7 (MCP3) but a notable absence of Th1 inflammatory chemokines early in infection ( 2 weeks). Such a chemokine profile suggests that the infection site might be restricted in its ability to recruit and/or activate antigen-specific effectors. Indeed, we demonstrated that IL-4- and IFN- $\gamma$-producing OVA-specific effectors differentially accumulate in the L. major ear. Although both effector cell types were recruited to an OVA/CFA immunization site, only IL-4 producers were found at the L. major infection site. Analysis of the L. major-specific cytokine repertoire revealed a similar site-specific restriction. Two weeks after infection, the dLN contained both anti-leishmania IL-4- and IFN- $\gamma$-producing cells. Strikingly however, the cytokine repertoire at the infected tissue site was limited to IL-4 and was mediated in part by local expression of CCL7. This narrowing of the cytokine repertoire at the L. major infection site could not be overcome by the administration of strong inflammatory signals (e.g., CpG and CFA), which indicates that L. major dominantly modifies the local milieu. Thus, an important control point in microbial pathogenesis may be mediated through the selective editing of the effector $T$ cell pool through differential recruitment or activation in the peripheral infected tissues.

\section{Results}

Restricted chemokine expression at the early L. major-infected dermis. To determine whether $L$. major infection results in a distinct inflammatory milieu at the site of infection, we first characterized chemokine expression in the infected ear dermis, the tissue site of natural infection $(23,28)$. Tissue was isolated 2 weeks after infection because this was the earliest time point at which $\mathrm{T}$ cell accumulation at the infection site was detected. A model protein antigen (OVA) and the standard immunization vehicles incomplete Freund's adjuvant (IFA) or CFA were used as positive controls to validate the extent of signals induced by $L$. major. The induction of chemokine expression in the ear was compared with that of PBS-injected controls (Figure 1). At this 2-week time point, CCL7 was reproducibly detected in the L. major-infected dermal tissue (Figure 1A). In contrast, there was a striking lack of proinflammatory chemokines (CXCL9, CXCL10, CCL3, and CCL5) in the early L. major-infected ear compared with IFA or CFA immunization. This restricted chemokine pattern was replaced by a broad spectrum of chemokine expression late in L. major infection (Figure 1B). Therefore, the early infection site is devoid of many chemoattractants that play a role in the recruitment of immune effectors at inflammatory sites.

Regulation of chemokine expression by L. major. L. major may simply fail to induce inflammatory signals or actively inhibit their expression. Therefore, we determined whether the provision of strong inflammatory mediators (e.g., CpG and LPS) could overcome the local L. major effect on chemokine expression. Mice were infected with $L$. major, and 2 weeks later the infection site was challenged with $\mathrm{CpG}$. Chemokine expression was measured from whole-ear extracts 30 hours after $\mathrm{CPG}$ challenge and compared with noninfected ears challenged with CpG. In support of an active downregulation of inflammatory signals, $\mathrm{CPG}$-induced chemokine expression was selectively attenuated in the L. major-infected ear (Figure 2A). L. major inhibited CPG induction of a number of chemokines and of the cytokines IL-10 and IL-12. In contrast, CCL7 continued to be predominantly expressed in the L. major-infected ear following CPG challenge (Figure 2A). To determine whether L. major mediated its effect through a direct action on the infected host cell, primary macrophage cultures were infected with $L$. major and subsequently exposed to CpG. L. major potently inhibited CpG induction of a number of proinflammatory chemokines (Figure 2B) but strongly induced the expression of CCL1 and to a lesser extent CCL7 (2-fold) (Figure 2B). CPG induction of IL-12 was also inhibited in L. majorinfected macrophages (Figure 2B, right), as previously described for LPS $(27,29)$. L. major also inhibited LPS-induced chemokine expression (data not shown). No inhibition of CpG-induced chemokines was observed in macrophage cultures exposed to soluble leishmania antigens (SLA) (data not shown). These in vitro studies suggest that the modulation of chemokine expression is in part a direct action of L. major within the infected macrophage. Differences between our in vitro and in vivo studies, particularly with respect to the induction of CCL7, may indicate indirect effects of L. major on noninfected cells types at the infection site.

Selective recruitment of cytokine effectors to the early L. major-infected dermis. The restricted chemokine expression pattern at the $L$. major infection site 2 weeks after infection suggests that the recruitment of antigen-specific $\mathrm{T}$ cells to this tissue site might be similarly restricted. CCL7 was expressed in the dermal tissue and has pleiotropic immune effects due to binding to multiple receptors (CCR1, CCR2, and CCR3) on monocytes, DCs, and T cells (30). However, one of the receptors, CCR3, is differentially expressed by Th2 cells and also by eosinophils and basophils (21). Conversely, the Th1-attracting chemokines CXCL10 (IP10), CCL3 (MIP1 $\alpha$ ), and CXCL9 (MIG) were all underrepresented at the infection site compared with CFA immunization. Although receptors for CCL5 are expressed on both Th1 (CCR5) and Th2 (CCR3) effectors, functionally RANTES also appears selective for Th1 recruitment (31) (S.D. Katzman, unpublished observations) and was also not upregulated by L. major infection. Thus, the early L. major infection site may differentially recruit/retain Th1 and Th2 effectors. Direct analysis of cytokine mRNA expression at the L. major-infected and OVA/CFA-immunized ears supported this notion: IL- 4 and IFN- $\gamma$ were detected in the OVA/CFA-immunized ear but a restriction toward IL-4 in the L. major-infected ear was observed at 2 weeks (Figure 2C).

To formally test the recruitment potential of the L. major site, we evaluated the homing of a cohort of OVA-specific effector cells to a L. major infection site (Figure 3A). The use of OVA/CFA-primed cells enabled us to directly assess the influence of $L$. major in the infected dermis distinct from the effects of $L$. major on the priming of the cytokine repertoire in the LN. Mice were injected in one ear with L. major and in the other ear with OVA/CFA. Two weeks after infection, the mice were challenged in the L. major-infected tissue with OVA protein. Thirty hours after challenge, OVA-specific effector cells were measured in the L. major-and OVA/CFA-infected ears with an antigen-specific (peptide OVA [pOVA], MHC Class II restricted) enzyme-linked immunospot (ELISPOT). This short time point was used to test the local accumulation of preexisting effectors rather than measuring new effectors primed following OVA challenge. Indeed, OVA challenge in the L. major-infected ear led to an increase 

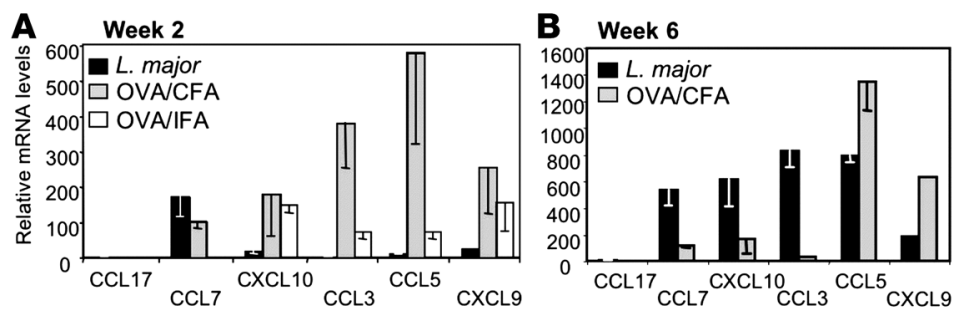

Figure 1

Chemokine expression in the ear dermis following $L$. major infection. mRNA was isolated from whole ear tissue of BALB/c mice 2 weeks (A) or 6 weeks (B) after L. major infection (black bars) or after OVA/CFA (gray bars) or OVA/IFA (white bars) immunization and chemokine expression measured by real-time RT-PCR. Values were calculated relative to PBS-injected ear tissue and calibrated to HPRT. Data are representative of results from at least 3 independent experiments.

in the total number of $\mathrm{CD} 4^{+} \mathrm{T}$ cells, $1,307 \pm 644(n=4)$ more cells per ear, over the 30-hour time frame. The dLN of the OVA/CFA immunized ear contained both IL-4- and IFN- $\gamma$-producing cells on recall with a Class II-restricted OVA peptide (Figure 3B, left). Similarly, both OVA-specific IL-4 and IFN- $\gamma$ producers were detected at the OVA/CFA dermal injection site (Figure 3B, right). Remarkably however, accumulation of OVA-specific cells in the OVA-challenged L. major infection site was dramatically skewed toward IL-4 (Figure 3B, right). There was the possibility that the restriction to OVA-specific IL-4-producing cells in the L. major-infected ear was driven not by the pathogen itself but by the nature of the OVA challenge, i.e., the use of PBS rather than a strong adjuvant. To test this, 2 weeks after mice were infected/immunized as shown in Figure 3A, their L. major-infected ears were challenged with OVA/CFA, and the accumulation of OVA-specific cells was measured 30 hours later. As for the OVA/PBS challenge, OVA-specific IL-4 producers dominated the OVA response in the L. major-infected ear with few IFN- $\gamma$ producers detected (Figure 3C). Thus, the restricted immune response at the L. major infection site remained dominant despite the presence of a strong adjuvant. These data, derived from an independent cohort of OVA/CFA primed effectors, establish that the early L. major-infected tissue is dominant and inherently unable to support the accumulation of IFN- $\gamma$ producers independent of priming conditions and antigen specificity.

It could be argued that $L$. major infection in one ear has systemic effects on priming of OVA-specific $T$ cells, which modifies their recruitment to the L. major-infected ear. To eliminate the effects of $L$. major on the priming of the OVA $\mathrm{T}$ cell response, $\mathrm{T}$ cells were primed in the absence of L. major infection and adoptively transferred to L. majorinfected recipients. Mice were immunized with OVA/CFA in the ear dermis, and $\mathrm{CD} 4^{+} \mathrm{T}$ cells were isolated from the $\mathrm{dLN} 2$ weeks later. In parallel, the ears of TCR $\mathrm{C}^{-/-}$mice were infected with $L$. major or immunized with OVA/CFA to serve as recipients of the OVA-primed $\mathrm{CD}^{+} \mathrm{T}$ cells. Donor $\mathrm{CD}^{+} \mathrm{T}$ cells contained both IL- 4 and IFN- $\gamma$ OVA-specific effectors (IL-4/IFN- $\gamma$ of 0.92 ) by ELISPOT. OVA-primed $\mathrm{CD}^{+} \mathrm{T}$ cells were transferred to the recipient mice, and their accumulation in the OVA-challenged ear tissue was determined 30 hours after cell transfer by ex vivo pOVA-specific ELISPOT (Figure 4). A mixture of both IL-4- and IFN- $\gamma$-producing OVA-specific CD $4^{+} \mathrm{T}$ cells was found in the OVA/CFA-immunized ear, but, again, there was a strong bias toward IL-4 producers at the L. major infection site (Figure 4A). In all experiments, the increased IL-4/IFN- $\gamma$ ratio in the ear represented the presence of IL-4 producers but the lack of IFN- $\gamma$ producers (e.g., OVA-challenged $L$. major ear: 77 IL-4 spots and 2 IFN- $\gamma$ spots). These results clearly demonstrate that $L$. major has a modulatory role on $\mathrm{CD} 4$ recruitment/retention at the local infection site distinct from the effects on the generation of the $\mathrm{T}$ cell cytokine repertoire in the dLN. The transfers isolate differences in CD4 cell accumulation at the infection site; in the intact animal, cytokine production from other immune sources may also be differentially regulated in the infected ear in a CD4-dependent or -independent fashion. In addition, the selective accumulation of effectors occurred in the infected host independent of $\mathrm{T}$ cells, which suggests that $L$. major modifies innate or nonhematopoietic cell types in the dermis to establish the restricted milieu.

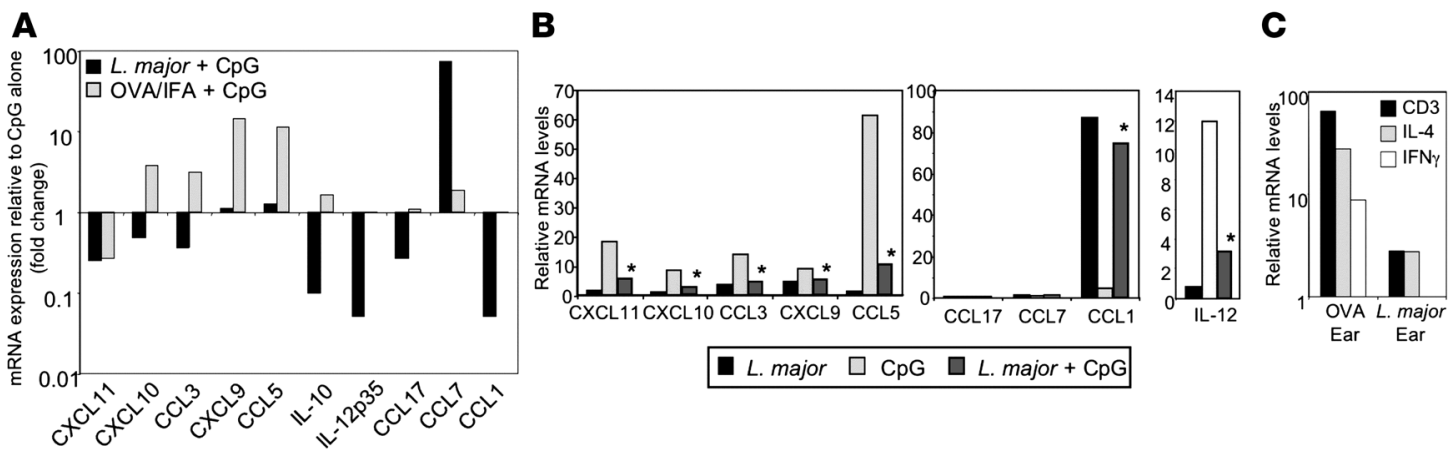

Figure 2

Dominant effect of $L$. major on chemokine expression. (A) BALB/c mice were infected with $L$. major, injected with OVA/IFA, or untreated. Two weeks later, mice were challenged with $\mathrm{CpG}$ for 30 hours, and mRNA was isolated from whole ear tissue for real-time RT-PCR analysis of chemokines and cytokines. Values are expressed as fold change over CpG-challenged ears in the absence of infection/immunization and relative to unmanipulated ears. Data are representative of 2 independent experiments. (B) BALB/c macrophages were challenged with CpG 4 hours after L. major infection, and chemokine expression was measured 24 hours later. mRNA levels by real-time RT-PCR were normalized to uninfected macrophages. ${ }^{*} P \leq 0.05$ for changes in chemokine expression on $\mathrm{CpG}$ challenge in the presence or absence of $L$. major by Student's $t$ test. Data are representative of results from 3 independent experiments. (C) CD3 and cytokine mRNA levels 2 weeks after $L$. major infection or OVA/CFA immunization in BALB/c mice. Values were calculated relative to PBS-injected ear tissue and calibrated to HPRT. Data are representative of results from 3 independent experiments. 
A

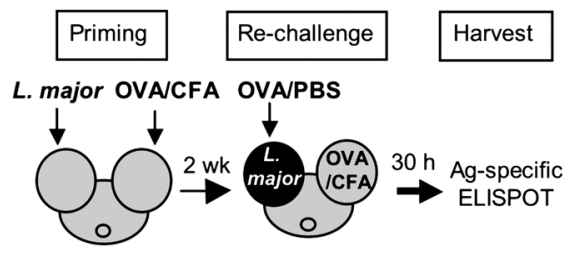

B

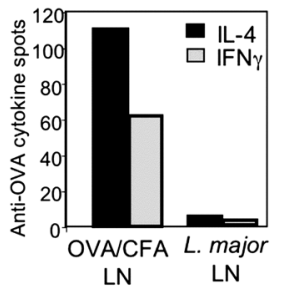

C

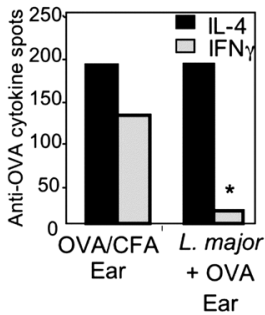

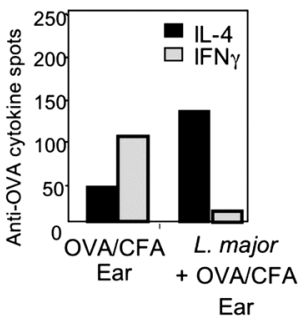

Figure 3

Selective recruitment of cytokine effectors to the early L. major-infected ear dermis. (A) Experimental design of OVA-specific recruitment studies. (B) Two weeks after infection/immunization of BALB/c mice, OVA-specific cytokine production was determined after in vitro restimulation with $5 \mu \mathrm{M}$ pOVA by ELISPOT (antigen-specific spots $/ 10^{6}$ for LN and total spots/ear). Left: OVA-specific cytokine response in the dLN from the OVA/ CFA immunized ear and from the LN draining the L. major-infected ear. Right: OVA-specific cytokine response in the ear of mice 30 hours after challenge with OVA protein, 2 weeks after infection of ear pinna with L. major or immunization of ear tissue with OVA/CFA. Fewer than 10 OVAspecific cytokine-secreting cells in the $L$. major-infected ear tissue were detected before OVA challenge. Data are representative of results from 4 comparable experiments. ${ }^{*} P=0.008$ for differences in IL-4/IFN- $\gamma$ ratio between OVA/L. major-infected ear tissue and OVA/CFA-immunized ear tissue as determined by Student's $t$ test. Similar results were obtained when C57BL/6 mice were used in the recruitment studies as in A. (C) $\mathrm{BALB} / \mathrm{c}$ mice were treated as in A, except the L. major-infected ear tissue was challenged with OVA/CFA, and OVA-specific recruitment to the ear was -analyzed as in B. Data are representative of results from 3 independent experiments.

Regulatory $\mathrm{T}$ cells clearly play a modulatory role in the IFN- $\gamma$ response to L. major (32). Our adoptive transfers to T cell-deficient hosts suggests that tissue resident Tregs are not essential for the restrictive milieu (Figure 4A). However, $\mathrm{CD} 4^{+} \mathrm{CD} 25^{+} \mathrm{T}$ cells can be recruited to the dermal site early in infection (32), which raises the possibility that Tregs in the cell inoculum may contribute to the restricted cytokine phenotype at the infection site. Therefore, we compared the accumulation of IL- 4 and IFN- $\gamma$ producers at the L. major site following the adoptive transfer of OVA-primed unfractionated $\mathrm{CD}^{+}$cells or $\mathrm{CD} 4{ }^{+}$cells depleted of $\mathrm{CD} 25^{+}$cells ("Tregdepleted," 99.2\% Foxp3-). The ratio of IL-4 to IFN- $\gamma$ producers in the infected ear following transfer of the $\mathrm{CD} 4^{+} \mathrm{CD} 25^{-} \mathrm{T}$ cells was identical to that observed with whole CD4s (Figure 4B). Thus, we found no evidence of a Treg requirement in the establishment of the early IL-4 restricted infection milieu.

Narrowing of the L. major-specific cytokine repertoire at the early L. majorinfected dermis. The restricted accumulation of third party OVA-specific effectors in the L. major infected dermis prompted us to analyze the available anti-L. major cytokine pool in the dLN and the active

\section{Figure 4}

Local control of cytokine responses within the infected ear dermis. (A) BALB/c OVA-primed CD4 T cells were isolated from the dLN 2 weeks after OVA/CFA immunization of mice and were transferred to $\mathrm{BALB} / \mathrm{c}$ TCR $\mathrm{C}^{-/-}$recipients injected with $L$. major or OVA/CFA 2 weeks before cell transfer. OVA-specific cytokine production by donor LN cells (before transfer) and ears of recipients was determined 30 hours after in vivo OVA protein challenge by in vitro antigen-specific ELISPOT. $P=0.024$ for the change in the ratio of cytokine-secreting cells between $L$. major-infected ears challenged with OVA and OVA/CFA-immunized ears. Data are representative of 3 independent experiments. (B) T cell transfers as in A with OVA-primed whole CD4 (black bars) or $\mathrm{CD}^{+}{ }^{+} \mathrm{CD} 25^{-}$(gray bars) cells isolated from the dLN and transferred to $L$. major-infected or OVA/CFA-immunized BALB/c TCR $\mathrm{C}^{-/-}$ recipients 2 weeks after infection or immunization. OVA-specific cytokine production by donor LN cells and recipient ears 30 hours after in vivo OVA protein challenge by in vitro antigen-specific ELISPOT. Data are representative of 2 independent experiments. cytokine pool at the infection site during the early stages of infection. $\mathrm{LN}$ and ear cell suspensions were isolated from tissues 2 weeks following L. major infection in the ear dermis (32), and the total number of cytokine-producing cells was analyzed by ELISPOT following in vitro restimulation with SLA. Despite a skewing in favor of IL-4-producing cells early after $L$. major infection $(33,34), L$. major-specific IFN- $\gamma$ producers were easily detected in the $\mathrm{dLN}$ early after infection (Figure 5, A and F). LN cytokine production, determined by ELISPOT, was mainly $\mathrm{CD}^{+}{ }^{+} \mathrm{T}$ cell-dependent, because both IL- 4 and IFN- $\gamma$ production were severely attenuated in the presence of an MHC Class II blocking antibody (data not shown). We used an established in vitro culture system (35) to further assess the phenotype of the anti-leishmania effectors in the dLN at this early infection time point. In vitro SLA stimulation of dLN cells from 2-week L. major-infected mice led to proliferation of both $\mathrm{CD}^{+}$and $\mathrm{CD}^{+} \mathrm{T}$ cells and highlighted that $\mathrm{CD}^{+} \mathrm{T}$ cells, and to a lesser extent $\mathrm{CD}^{+} \mathrm{T}$ cells, were primed for IL-4 and IFN- $\gamma$ production (Figure 5B).

In stark contrast, only IL-4 producers were detected at the early infection site (Figure 5A). This cytokine profile on 6-hour anti-
A

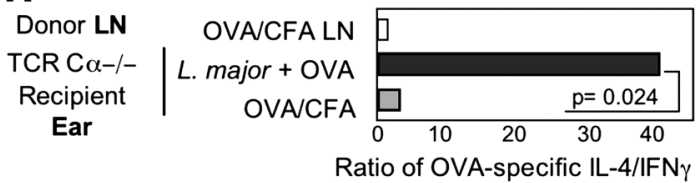

B

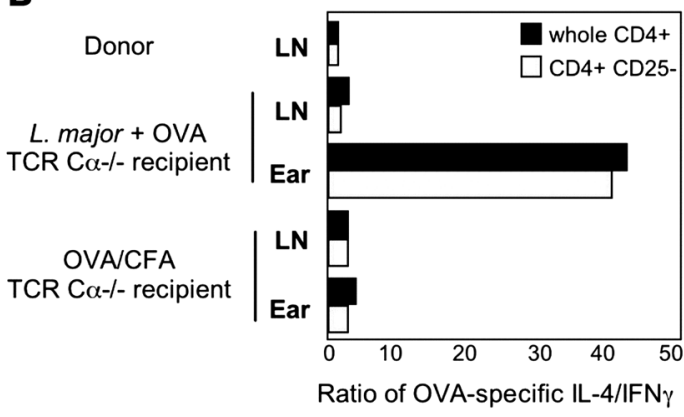


A IFN

Week 2

LN
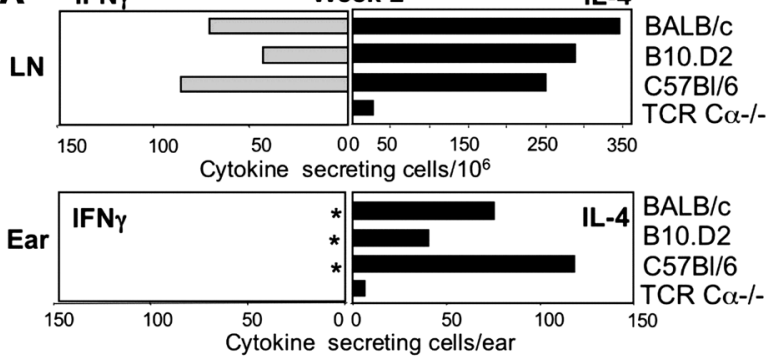

C

\begin{tabular}{|c|c|c|c|}
\hline Exp & \# CD4+ & IL-4 & IFN $\boldsymbol{\gamma}$ \\
\hline 1 & 2700 & 112 & 4 \\
\hline 2 & 1540 & 87 & 1 \\
\hline 3 & 1464 & 69 & 2 \\
\hline 4 & 3252 & 135 & 0 \\
\hline
\end{tabular}

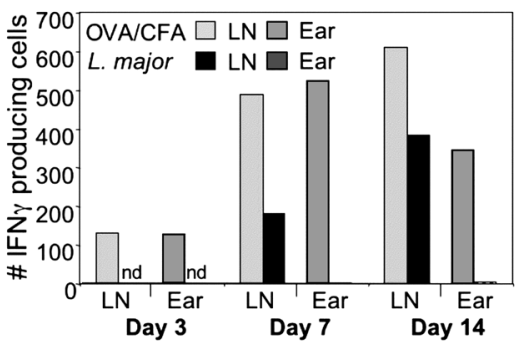

D

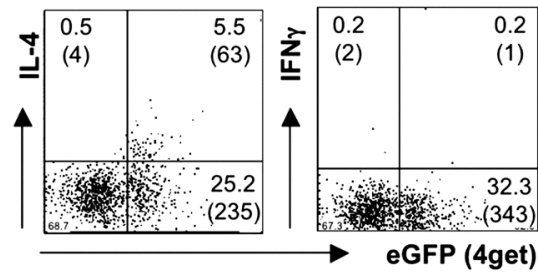

G
B

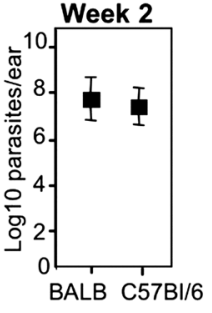

E

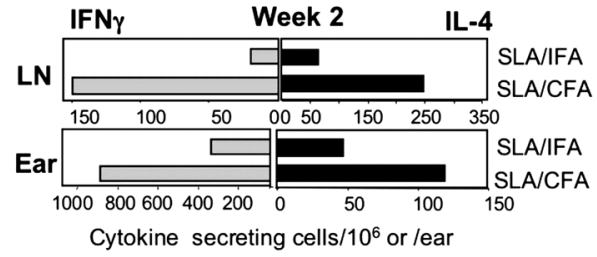

H

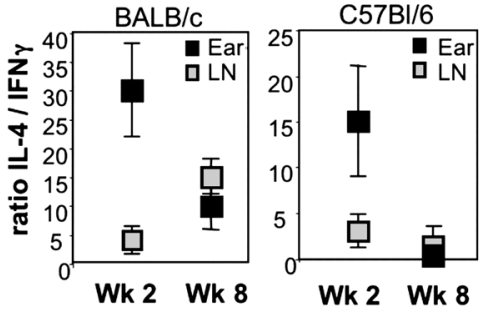

Figure 5

Restricted cytokine responses at the site of early L. major infection. (A) Ear and dLN cell suspensions from mice 2 weeks after infection with L. major were stimulated for 6 hours with SLA in vitro, and cytokine-producing cells were determined by ELISPOT. Each bar represents a pool of 4 infected mice. ${ }^{*} P$ values for the increase in the ratio of IL-4/IFN- $\gamma$ in the infected ear compared with the dLN as determined by Mann-Whitney $U$ test: $P=0.002$ BALB/c mice, $n=12$ experiments; $P=0.01$ for B10.D2 mice, $n=6$ experiments; and $P=0.002$ for C57BL $/ 6$ mice, $n=6$ experiments. Right: Parasite load in ear 2 weeks after infection. (B) CFSE-labeled LN cells from BALB/c mice were cultured for 72 hours with SLA and cytokines measured by intracellular cytokine staining. Numbers represent the percentage of $L$. major-specific cytokine-secreting cells. (C) Total CD4+ $\mathrm{T}$ cell numbers and $L$. major-specific cytokine-producing spots in the ear of BALB/c mice 2 weeks after $L$. major infection. (D) Ex vivo production of IL-4 and IFN- $\gamma$ measured by cytokine capture assay, 2 weeks after L. major infection of BALB 4get mice. Plots gated on CD4+ $\mathrm{T}$ cells. The numbers in quadrants represent the percentage and numbers (in parentheses) of CD4+ $\mathrm{T}$ cells per ear. Background staining was $0.01 \%-0.2 \%$. Data are representative of 3 separate experiments. (E) BALB/c mice were immunized with SLA in the ear dermis, and 2 weeks later cytokine production was assayed as in $\mathbf{A}$. Data are from 1 of 3 comparable experiments. $(\mathbf{F})$ BALB/c mice were injected with OVA/CFA or L. major. Antigen-specific cytokines were assayed as in A. Results are representative of at least 3 individual experiments. Results were similar in L. major-infected C57BL/6 mice. (G) BALB/c LACK-specific TCR Tg+ mice were infected with L. major, and cytokines were analyzed 2 weeks later as in $\mathbf{A}$. ${ }^{*} P \leq 0.05$ for the difference in IFN- $\gamma$-producing cells in the ear and the dLN as determined by Student's $t$ test. (H) Ratio of IL-4/IFN- $\gamma-$ producing cells in BALB/c and C57BL/6 mice from the dLN and ear and determined as in A. Data are the mean \pm SD of 4 experiments.

gen-specific restimulation in vitro closely mimicked that measured directly ex vivo by real-time RT-PCR of the whole ear tissue (Figure 2C). IL-4 production in the ear was dependent on CD4 $\mathrm{T}$ cells because few cytokine-secreting cells were detected following ex vivo depletion of $\mathrm{CD}^{+} \mathrm{T}$ cells from ear suspensions (data not shown) or from L. major-infected TCR C $\alpha^{-/-}$mice (Figure 5A). Although the total number of $\mathrm{CD} 4^{+} \mathrm{T}$ cells in the ear at this early infection time point was small and variable between experiments (Figure 5C), using IL-4 reporter mice (4get; ref. 36) we were able to clearly detect an $L$. major-specific eGFP $\mathrm{IL}^{+} 4^{+} \mathrm{CD} 4^{+} \mathrm{T}$ cell population directly ex vivo but no IFN- $\gamma^{+} \mathrm{CD}^{+} \mathrm{T}$ cells (nor IL-2 producing cells; data not shown) from the infection site (Figure 5D). A non-T cell population was also $\mathrm{eGFP}^{+}$in the ear at a similar frequency to the $\mathrm{GFP}^{+} \mathrm{CD} 4 \mathrm{~T}$ cells, but only $6 \%$ of the non-T $\mathrm{eGFP}^{+}$ cells were making IL-4 ex vivo compared with $20 \%$ of the eGFP ${ }^{+}$
$\mathrm{CD}^{+} \mathrm{T}$ cells. Few CD8 ${ }^{+} \mathrm{T}$ cells were present in the ear 2 weeks after infection (average: $120 \mathrm{CD}^{+} \mathrm{T}$ cells per ear), and cytokine production was undetectable.

The presence of IL-4 but not IFN- $\gamma$ producers at the infection site was highly significant in all mouse strains tested (Figure 5A). At this early time point, the parasite load in the ear dermis was comparable between strains (Figure 5A, right). Importantly, as with the OVA immunizations, the ear dermis itself did not inherently skew effector cell accumulation because both IL- 4 and IFN- $\gamma$ producers were detected in the ear on immunization with Leishmania antigens in IFA or CFA (Figure 5E).

Unlike our studies with OVA immunization, in which a similar frequency of IL-4 and IFN- $\gamma$ producers was generated in the dLN (Figure 3 ), the total number of IFN- $\gamma$ producers in the dLN in response to L. major infection (at 2 weeks) was always lower than that of IL-4 
A
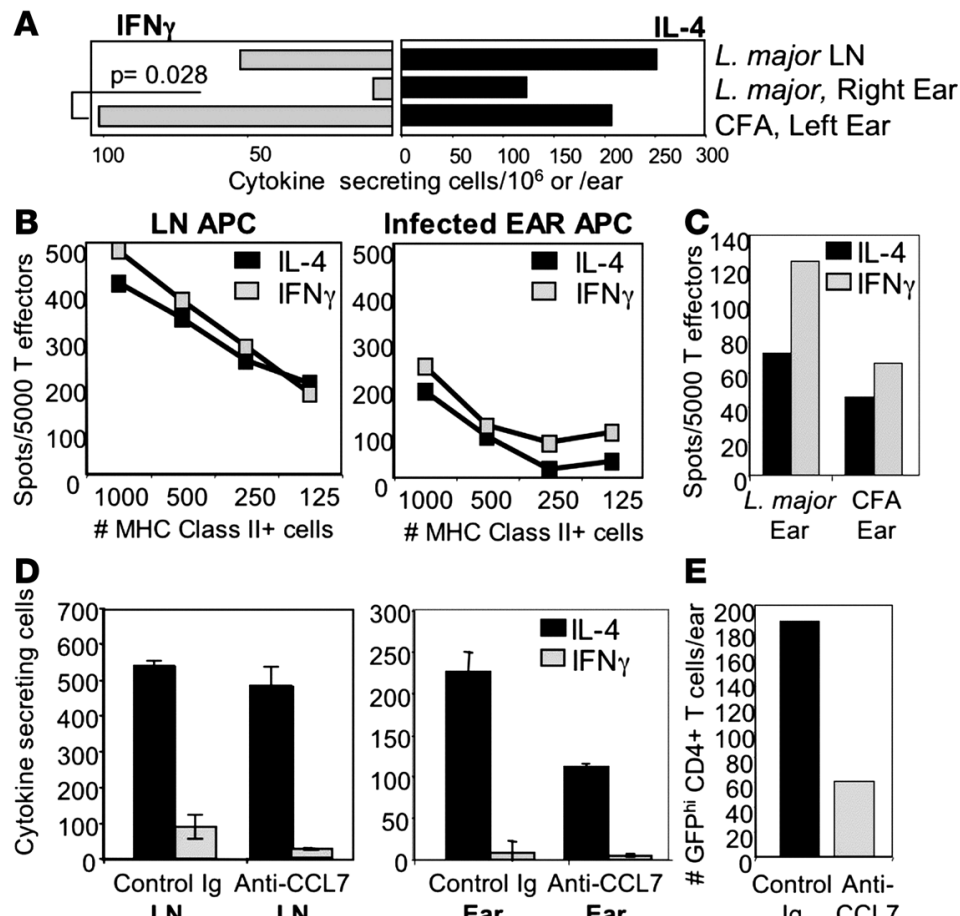

\# MHC Class II+ cells

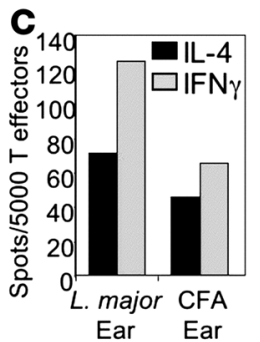

E

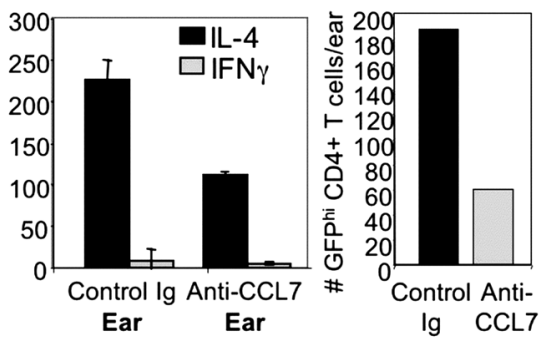

\section{Figure 6}

Site-specific and CCL7-dependent accumulation of distinct cytokine-producing effector T cells. (A) BALB/c mice were infected with $L$. major in the right ear and injected with CFA in the left ear. L. major-draining $L N$ and $L$. major or CFA ear cell suspensions were stimulated 2 weeks later with SLA for cytokine ELISPOT. Data are representative of 3 comparable experiments. $P$ value by Mann-Whitney $U$ test. Similar results were observed in C57BL/6 mice. (B) APCs from uninfected LN (left) and from ear tissue of BALB/c mice 2 weeks after infection with $L$. major (right) were used to restimulate D011.10-primed Th1 and Th2 effector cells, and cytokines were measured by antigen-specific ELISPOT with $5 \mu \mathrm{M}$ pOVA 323-339 for 6 hours (cytokine production from T cells alone and APCs alone subtracted). (C) Restimulation of in vitro primed effectors with APC from ears of 2-week L. major-infected or CFA-immunized $B A L B / c$ mice. APC numbers were normalized to $I^{d+}$ cells for B and C. Data are representative of 3 comparable experiments. (D) An anti-CCL7 polyclonal or control Ab (250 $\mu \mathrm{g} / \mathrm{ear})$ was injected directly into $L$. major-infected dermis of BALB/C 4 get mice 2 weeks after infection. Three days after antibody treatment, cells were isolated from the dLN and the ear and restimulated with SLA. The number of IL-4-producing cells was then determined by ELISPOT. Cytokine-producing cells $/ 10^{6}$ for $L N$ or per ear; $n=6$ mice per group. (E) Number of eGFPhi CD4 ${ }^{+} \mathrm{T}$ cells in the dermis after $\mathrm{Ab}$ treatment of L. major-infected ears by FACS. Data in $\mathbf{D}$ and $\mathbf{E}$ are representative of 2 comparable experiments. producers. This finding suggests that differences at the infection site may have been due to differences in effector cell frequency. We performed a kinetic analysis of the frequency of IFN- $\gamma$ producers during the early phases of $L$. major infection and compared that with the IFN- $\gamma$ response on OVA/CFA immunization. The total number of IFN- $\gamma$ producing cells was calculated after a 6 -hour in vitro ELISPOT with SLA or POVA (Figure 5F). As expected, IFN- $\gamma$ producers were detected early after OVA/CFA immunization (day 3 ) in the $\mathrm{dLN}$, whereas detection of $L$. major-specific IFN- $\gamma$ producers lagged behind and was first detected on day 7. Despite this delay in the kinetics of the L. major response, importantly, the magnitude of the L. major-specific LN IFN- $\gamma$ response was not dramatically different from that of the OVA/CFA LN. In contrast, whereas OVA-specific IFN- $\gamma$ producers were detected in the OVA-immunized ear tissue throughout the time course (even at day 3 when the LN frequency of IFN- $\gamma$ producers was low), no IFN- $\gamma$ producers were detected in the L. major-infected ear tissue in the first 3 weeks despite appreciable numbers in the LN. Therefore, the number of $L$. major-specific LN IFN- $\gamma$ producers per se does not explain why they were not detected in the infected ear. Our recall of OVA-specific T cells at the immunization site used the MHC Class II restricted peptide OVA $323-339$; CD4-dependent responses were mixed (IL-4 and IFN- $\gamma$ ) in the OVAimmunized ear. In addition to this CD4 component, we could not rule out a differential role in vivo for other immune cells, such as $\mathrm{CD}^{+} \mathrm{T}$ cells, in shaping the cytokine response in the ear following L. major versus OVA immunization. Nonetheless, this kinetic study clearly demonstrates that the IFN- $\gamma$ response in the $\mathrm{LN}$ is not well represented at the L. major infection site and that this LN/tissue discrepancy is not observed for responses to protein antigens for which the IFN- $\gamma$ response in the $\mathrm{LN}$ and injection site is comparable.

To further address the question of low IFN- $\gamma$ T cell numbers and the balance between IL- 4 and IFN- $\gamma$ production at the 2 -week infection time point, we used an L. major-specific TCR transgenic mouse approach to considerably increase the available anti-L. major IL- 4 and IFN- $\gamma$ producers in the dLN (Figure 5G). L. major infection led to a 10- and 2.5-fold increase in IFN- $\gamma^{+}$and IL- $4^{+}$cells, respectively, compared with non- $\mathrm{Tg}^{+}$mice at 2 weeks, which resulted in a $1: 1$ ratio of IL- 4 and IFN- $\gamma$ producers in the dLN. Despite a larger IFN- $\gamma$-producing pool and equivalent IL- 4 and IFN- $\gamma$ frequencies, only IL-4 was detected at the local $L$. major infection site in TCR transgenic mice (Figure 5G). Therefore, the inability to detect IFN- $\gamma$ producers in the ear tissue does not appear to be a consequence of the generation of lower numbers of IFN- $\gamma$ producers or the presence of a predominant IL-4 response in the dLN.

Late in the infection (week 8), the cytokine response in the infected tissue more closely resembled that of the dLN (Figure $5 \mathrm{H}$ ). Thus, early in the infection, despite a mixed IL- 4 and IFN- $\gamma$ T cell repertoire in the $\mathrm{dLN}$, the cytokine response at the nonlymphoid infection site was restricted to IL-4. Similar results were obtained with infection of either L. major promastigotes or amastigotes (data not shown) and was independent of the genetic background of the host (Figure 5, A and $\mathrm{H}$ ). This finding suggests that L. major itself shapes the dermal environment early in the infection, possibly to evade leishmaniacidal IFN- $\gamma$ producers.

Site-specific accumulation of distinct cytokine-producing effector T cells. Our cytokine data revealed a narrowing of the cytokine repertoire from the dLN to the infection site. The failure to detect IFN- $\gamma$ production at the L. major infection site could have been due to an intrinsic defect in the homing potential of the LN-derived IFN- $\gamma$ effectors early in the infection. Therefore, to directly test the trafficking potential of the L. major-primed IFN- $\gamma$ producers, we determined whether they could home to an inflamed dermis in the absence of local L. major influences. Mice were injected in one ear with $L$. major and in the other ear with CFA alone; cytokine responses were measured 2 weeks later. Strikingly, both IFN- $\gamma$ and IL- 4 producing $L$. major-specific cells accumulated in the CFA inflamed der- 

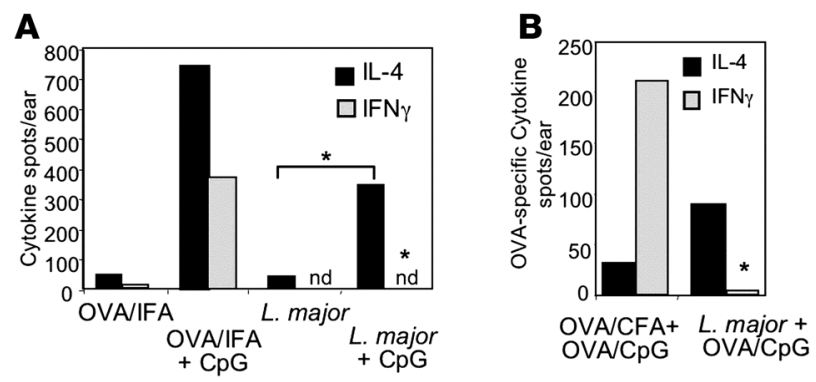

Figure 7

Dominant effect of $L$. major on the local tissue microenvironment. (A) BALB/c mice were injected with OVA/IFA in one ear and infected with L. major in the contralateral ear. Two weeks later, mice were challenged with $\mathrm{CpG}$ for 30 hours and cells were isolated for detection of cytokine production ex vivo by ELISPOT. ${ }^{*} P=0.04$ for difference in increase in the number of IL-4-producing cells between the ear tissue infected with $L$. major and challenged with $C p G$ and the ear tissue infected with L. major alone (Student's $t$ test); $P \leq 0.03$ for the differences between IFN- $\gamma$-producing cells in the ear tissue infected with $L$. major and challenged with $\mathrm{CpG}$ and ear tissue immunized with OVA/IFA and challenged with $\mathrm{CpG}$ (Student's $t$ test). nd, not detectable. (B) OVA-specific cells in L. major-infected or OVA/CFA-immunized ear tissue 30 hours after in vivo challenge with OVA/CpG determined by ELISPOT as in A. ${ }^{*} P \leq 0.03$ for the difference in OVA-specific IFN- $\gamma$-producing cells between ear tissue infected with $L$. major and challenged with OVA/CpG and OVA/CFA plus OVA/CpG-immunized ear (Student's $t$ test). Data are representative of at least 3 independent experiments.

mis, but only IL-4 production was detected in the L. major-infected ear (Figure 6A). Importantly, although there have been reports of parasites homing from the infection site to the contralateral noninfected ear early in L. major infection (37), in our system, by limiting dilution culture analysis, we were unable to detect $L$. major in the contralateral CFA site. Thus, L. major priming in the LN does not alter the intrinsic potential of the $L$. major-specific IFN- $\gamma$ producers to exit the LN, enter sites of inflammation, or retain effector function. The ability of Leishmania-specific Th1 cells to home to CFAinduced inflammatory sites suggests that their failure to home to L. major-infected sites resides at the tissue site of infection.

L. major-specific IFN- $\gamma$ producers may fail to be recruited to the infection site or, in contrast, enter the ear but be functionally modified in the local milieu; these possibilities not being mutually exclusive. If IFN- $\gamma$ producers reach the infection site, they could be disabled there because of the lack of appropriate activation signals. To test the functional capacity of APCs at the L. major site, we isolated cells from L. major-infected and CFA-immunized ears and compared their ability to support IFN- $\gamma$ production with APCs from noninfected LNs using in vitro-primed $\mathrm{CD}^{+}{ }^{+}$effectors. Whereas the magnitude of cytokine production was lower when OVA was presented by APCs from the L. major-infected ear than from LN APCs, the L. major-infected ear tissue supported the production of both IFN- $\gamma$ and IL-4 (Figure 6B) similarly to the response with APCs from CFA ears (Figure 6C). Analogous results were seen after in vivo antigen loading of ear APCs by injection of OVA protein before the harvest of ear tissue and their use in in vitro stimulation cultures. In addition, neither the in vitro provision of SLA-pulsed "competent" APCs (T-depleted spleen or A20) to L. major-infected ear suspensions nor stimulation with PMA/ ionomycin revealed IFN- $\gamma$-producing cells in the ear extracts (data not shown). Thus, the L. major-infected ear environment is capable of activating Th1 effectors and does not appear to harbor cells with the potential for IFN- $\gamma$ production. This analysis of the activation potential of the ear milieu together with our analysis of cytokine and chemokine expression at the site of $L$. major infection supports a model of differential recruitment, rather then differential activation, of Th1/Th2 effectors at the early infection site.

CCL7 was the main chemokine upregulated 2 weeks after $L$. major infection. Its expression has been associated with a variety of disease states, including allergic inflammation, presumably because of CCR3 expression on eosinophils and Th2 cells (38). Interestingly, CCL7 also antagonizes CCR5 signaling (in response to MIP-1 $\beta$ and RANTES) and suppresses IL-12 $(39,40)$. Thus, CCL7 may potentiate the IL-4 restriction at the L. major site through positive enforcement of a Type 2 response or negative regulation of Th1 recruitment and/or activation. To directly address the contribution of CCL7 to the shaping of the restricted milieu at the L. major infection site, we blocked CCL7 activity with an anti-CCL7 Ab (41). The polyclonal anti-CCL7 Ab was previously shown to be highly specific for CCL7 after rigorous testing against a panel of 16 different chemokines (41). BALB/c 4get mice were infected with L. major; 2 weeks later, anti-CCL7 or control $\mathrm{Ab}$ was administered directly into the ear dermis. Three days after antibody treatment, cells were isolated from the ear, and the number of anti-leishmania IL-4- and IFN- $\gamma$ producing cells was determined by ELISPOT. CCL7 antibody treatment did not significantly alter the number of IL-4- and IFN- $\gamma$ producing cells in the dLN (Figure 6D, left). However, short-term blocking of CCL7 led to a 50\%-68\% reduction in the number of IL-4producing cells in the infected ear measured by ELISPOT (Figure 6D, right) and by FACS analysis of the number of $\mathrm{CD}^{+} \mathrm{GFPhi}^{\mathrm{T}}$ cells (Figure 6E). A similar reduction in the number of eosinophils was also observed in the ear following anti-CCL7 treatment, but not after treatment with control $\mathrm{Ab}$, as described previously (41). In contrast, blocking CCL7 did not increase the number of IFN- $\gamma$-producing $\mathrm{T}$ cells in the ear. Therefore, CCL7 expression appears to function through the recruitment/retention of IL-4 producers and not by negatively regulating IFN- $\gamma$-producing T cells.

Dominant effect of $L$. major on the local tissue environment. Our previous analysis of chemokine expression suggests that $L$. major plays an active role in inhibiting macrophage responsiveness to inflammatory stimuli (Figure 2). To determine whether this also held true for the restriction on the accumulation of cytokine-producing cells, we administered CPG to the ear tissue 2 weeks after infection with $L$. major and measured the accumulation of cytokine-producing cells 30 hours later. Once again, the 30 -hour time point was used to exclude the possibility of the recruitment of new effectors primed following the $\mathrm{CPG}$ challenge. Administration of CPG led to an overall increase in the frequency of both IL-4 and IFN- $\gamma$ producers in the OVA-immunized ear (Figure 7A). In stark contrast, only an increase in IL-4 producers was observed in the CPG-challenged L. major-infected ear (Figure 7A). A similar inability to disrupt the $L$. major milieu with respect to differential accumulation of effectors was observed with the administration of CFA to the L. major infection site at 2 weeks (Figure 3C). The results were further verified using the anti-OVA recruitment system (Figure 3); OVA-specific IL- 4 and IFN- $\gamma$ producers were recruited to an OVAimmunized ear following CPG challenge, but only OVA-specific IL-4 producers were detected in the CPG/OVA-challenged L. majorinfected ear tissue (Figure 7B). Thus, the presence of $\mathrm{CPG}$ was unable to override the L. major-induced restriction toward IL-4. 
Therefore, L. major appears to play a dominant role in the differential expression of chemokines and differential accumulation of IL- 4 and IFN- $\gamma$ producers in the early infection site, even in the face of strong inflammatory signals.

\section{Discussion}

Our data reveal an unexpected effect of $L$. major on $\mathrm{T}$ cell responses at the dermal infection site. Analysis of early chemokine expression suggests that $L$. major might modulate the ability of the tissue site to recruit or retain cytokine-producing $\mathrm{T}$ cell subsets. We directly tested this notion by analysis of the recruitment of OVA-specific effectors to the L. major infection site. Indeed, both IL-4 and IFN- $\gamma$ OVA-specific effectors were detected in the OVA/CFA-challenged ear, but only IL-4 producers were detected in the OVA-challenged L. major-infected ear tissue. Similarly, a mixed (IL-4 and IFN- $\gamma$ ) leishmania-specific cytokine response generated centrally (LN) following L. major infection of the ear dermis was narrowed at the peripheral infection site. Early in the infection (2 weeks), L. major-specific IL-4 and IFN- $\gamma$ producers were detected in the LN, but only IL-4 producers were measured in the infected dermis. The recruitment/retention of IL-4 producers was mediated in part by pathogen-induced CCL7. Thus, L. major appears to modify the local environment, which results in the selective accumulation of IL-4, but not IFN- $\gamma$, producers. The use of OVA-primed effectors enabled separation of the effects of $L$. major on $L N$ priming from the effects of $L$. major on the local accumulation of cytokine-producing cells in the infected dermis. Our results clearly demonstrate that $L$. major appears to have a distinct modulatory role in the local infection site, independent of the effects on the generation of the anti-leishmania $\mathrm{T}$ cell cytokine repertoire in the dLN. Additionally, the results strongly suggest that L. major regulates the differential recruitment of Th1 and Th2 effectors to the infected dermis early after infection.

The Sacks group (28) previously provided a detailed analysis of the kinetics of cytokine production in the L. major-infected dermis for the low dose "natural" infection model. An early IL-4-biased phenotype in the infected ear was also observed in this system for the first 2 weeks of infection, although the phenotype of the IL-4producing cells was not determined. Interestingly, unlike our observation of a distinct difference in the IL-4/IFN- $\gamma$ ratio between the dLN and the infection site early in the infection, in the low dose infection study, the cytokine phenotype in the ear closely mimicked that seen in the dLN. We cannot fully explain the differences between the 2 studies, but suggest that our experimental design revealed local restrictions on the cytokine response because of the more rapid generation and frequency of IFN- $\gamma$ producers in the dLN either through OVA/CFA priming or following infection with larger numbers of parasites. Indeed, both anti-leishmania-specific IL-4 and IFN- $\gamma$ producers were detected in the dLN 1 week after infection with $L$. major and coincided with the early IL-4 bias in the ear tissue (data not shown). In contrast, in the natural model of dermal infection, IFN- $\gamma$ producers were first detected in the dLN and ear much later, at 4 weeks. Our novel observations using the OVA-recruitment system (Figure 3) demonstrate that the infection site clearly modulates the recruitment of cytokine effectors. The OVA-recruitment system formally separates the effects of pathogens on priming and the effects of pathogens at the dermal site. Via this approach, it will be interesting to see whether this local regulation of the ear dermis operates when parasite numbers are low, either through infection with fewer parasites or during long-term parasite persistence in resistant mouse strains.
Remarkably, the restricted cytokine and chemokine response in the L. major-infected ear was dominant even in the face of strong inflammatory signals (Figures 2 and 7), which suggests active downregulation of the early innate response to microbial challenge. Such a milieu could be established through the manipulation of gene expression for a variety of inflammatory cytokines, chemokines, and adhesion molecules via the innate or nonhematopoietic compartments. Indeed, in vitro studies have revealed the reduced expression of numerous immune response, signaling, and cell physiological genes within macrophages following L. major infection $(26,42)$. Macrophage production and/or secretion of a number of inflammatory mediators (e.g., IL-1 $\beta$, IL-12, and NO) is inhibited by Leishmania infection, possibly through manipulation of NF-кB and MAPK signaling pathways (27, 43-45). Therefore, L. major could have strong local effects on the microenvironment that alter recruitment and/or retention signals for a variety of immune effectors. However, the induction and repression of inflammatory mediators by L. major during the course of infection in the dermis, in vivo, is less clear $(29,46-48)$. We showed quantitative differences in the induction of cytokines and chemokines at the site of infection in vivo, with L. major inducing little or no expression of a panel of inflammatory mediators that were induced following CFA immunization. Nonetheless, the dominant nature of the cytokineselective milieu predicts an active rather than a passive regulation of inflammation. Indeed, a number of Leishmania species have been shown to inhibit LPS-induced macrophage responses, including IL-12 and IL-1 $\beta$ production $(27,29)$. Additionally, the studies with L. major and CPG described herein showed that Leishmania infection can also modulate chemokine expression in the infected host cell. Similar active regulation of chemokine expression was observed in L. major-infected macrophages exposed to LPS (S.D. Katzman and D.J. Fowell, unpublished observations). Interestingly, L. mexicana

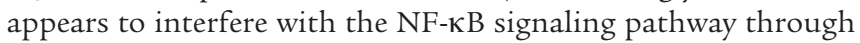
the activity of a parasite-derived cysteine peptidase (27). Such a mechanism may account for the dominant effect of $L$. major over CPG, CFA, or LPS, possibly through direct interference with common elements in the signaling pathways downstream of a variety of TLRs. Whereas CPG did not alter the L. major milieu with respect to the initial recruitment of IFN- $\gamma$ producers, administration of $\mathrm{CpG}$ during $L$. major infection or in vaccine settings has been shown to clearly enhance the clearance of parasites $(49,50)$. It is not clear whether this is due to enhanced generation of IFN- $\gamma$ producers in the dLN or to better recruitment to the site of infection. In addition, coadministration of CPG and L. major in the vaccine studies (49) may disrupt the competitive advantage of having L. major already resident in the macrophage, as in our experimental setup and in that of others studying the effect of LPS in L. major-infected macrophages and in natural infection.

Selective recruitment of effector T cells is an attractive possibility for the presence of IL-4 but not IFN- $\gamma$ producers in the L. majorinfected ear given the known differences in chemokine receptor and adhesion receptor expression by Th1 and Th 2 effectors and the chemokine profile expressed at the infected tissue site. Homing studies are complicated by the dynamics of recruitment and retention of antigen-specific T cells. Studies to track the initial entry of functionally marked $\mathrm{T}$ cell populations in live animals are underway using a combination of fluorescent cytokine reporters for function and transgenic expression of luciferase for localization. The requirement for tissue-specific expression of STAT1 and STAT6 for the trafficking of Th1 and Th2 effectors, respectively $(11,12)$, may 
contribute to the phenotype in the L. major-infected ear. Leishmania species have been shown to modulate a number of cytokines and chemokines (51); however, the predominance of CCL7 (MCP-3) expression early in infection has not been previously noted. Using a highly specific polyclonal anti-CCL7 Ab (41), transient local blockade of CCL7 at the infection site led to a marked reduction in the number of IL-4-producing cells (Figure 6, D and E) without a concomitant increase in IFN- $\gamma$ producers. Thus, pathogen-induced CCL7 appears, in part, to regulate the recruitment/retention of IL-4-producing cells in the L. major-infected ear dermis. The cellular source of CCL7 in the L. major infection setting remains to be elucidated. Many cell types have been shown to inducibly express CCL7, including monocytes, mast cells, epithelium, and endothelium (30). Interestingly, in a study of CCL7 expression in Schistosoma mansoni egg-induced lung granulomas, CCL7 was restricted to the blood vessels close to the granuloma and appeared to be positively regulated by IL-4 (41). Our preliminary studies found no induction of CCL7 following L. major infection of TCR C $\alpha^{-/-}$mice, which suggests that CCL7 expression is T cell-dependent. Analogous to the granuloma study (41), T cells may amplify CCL7 expression via IL-4 production. Interestingly, vaccination with a Leishmania antigen in combination with CCL7 induced a nonprotective Th2 response (52); thus, CCL7 may serve as a useful target for the manipulation of type 2 responses in the skin.

Regulation of effector $\mathrm{T}$ cell recruitment and/or activation at the infection site may also be modulated by cytokines. IL-10 appears to be a key effector molecule in the regulation of antiL. major responses by Tregs or IFN- $\gamma^{+} / \mathrm{IL}-10^{+} \mathrm{Th} 1$ cells $(32,53)$. We found no evidence of a requirement for Tregs in our system (Figure 4), but IL-10 from non-T cell sources may also contribute to the infected microenvironment. In the absence of IL-10, mice undergo sterile cure possibly because of the generation and/or recruitment of a more potent Th1 response (54). Thus, local IL-10 production may contribute to the differential accumulation of IL- 4 and IFN- $\gamma$ producers at the L. major infection site. However, we were unable to detect elevated IL-10 expression in whole ear extracts 2 weeks after infection. Therefore, the role of IL-10 in the differential accumulation of cytokine producers shown here remains to be formally tested with adoptive transfers into infected T cell-deficient IL-10-/mice. Alternatively, the lack of IL-12 induction with L. major infection may also alter the recruitment or potency of IFN- $\gamma$ production at the infection site. IL-12 was shown to regulate chemokine expression in the dLN of L. major-infected mice (55) and appears to be required not just for the induction of a Th1 phenotype but also for the maintenance of Th1 effector function in a number of disease settings, including L. major (56-59). IL-12 was not detected in the dermal tissue 2 weeks after infection with $L$. major, and its induction following $\mathrm{CPG}$ administration was strongly inhibited (Figure 2A). Therefore, it remains possible that the absence of IL-12 at the tissue site may contribute to the in vivo phenotype described herein. In addition, the dominant IL-4 response in the ear may also contribute to the restricted milieu either through well-established roles in the downregulation of macrophage function and modulation of chemokine expression or perhaps through the direct inhibition of Th1 function (60). Although the CD4-dependent IL-4 response dominates in the early lesion, it is clearly not essential for the restrictive L. major-infection milieu, because this can be established in T cell-deficient hosts (Figure 4). It will be interesting to determine the relative role of IL- 4 produced by $\mathrm{T}$ and non- $\mathrm{T}$ cell sources in regulating the infected tissue environment.
Although there does not appear to be a general defect in the capacity of the infection site to support Th1 effector function (Figure 6, $\mathrm{B}$ and $\mathrm{C}$ ), we cannot rule out the possibility that IFN- $\gamma$ producers are recruited to the infected site but are subsequently functionally modified in the L. major-infection milieu. Ex vivo comparison of L. major-infected and-uninfected APCs from the infection site may help to distinguish between local and systemic effects within the ear itself. Recent observations show that chemokines aid in $\mathrm{T}$ cell activation (61); therefore, the lack of Th1 chemokines may also affect the function of Th1 effectors at the site. A number of recent studies have identified distinct biochemical signal requirements for the initial acquisition and subsequent execution of effector function, at least for Th2 cells (62-65). Thus, control of secondary $\mathrm{T}$ cell activation signals in the infected tissue may play an important modulatory role in the anti-pathogen response. Distinct signals for Th1 effector function are less well characterized $(66,67)$. In the case of $L$. major, IFN- $\gamma$ producers may traffic to the ear similarly to IL-4 producers, but fail to receive the appropriate activation signals for effector function. The presence of antigen appears to promote the accumulation of effectors in peripheral tissues (68), possibility through signals that stop further migration (69); however, the signals that support effector cytokine production in the tissue are not well characterized. In studies on the effector cell types in the schistosome granuloma, IFN- $\gamma$ producers were present in the granuloma milieu but were functionally silent, but could be activated to secrete IFN- $\gamma$ if stimulated ex vivo (20). Using similar experimental approaches to the schistosome study, we attempted to reveal IFN- $\gamma$ potential in the L. major-infected ear by provision of competent antigen-presenting cells or strong activation signals (PMA/Ionomycin) ex vivo. However, we were unable to find significant numbers of quiescent anti-leishmania CD $4^{+}$IFN- $\gamma$-producing cells in the L. major-infected ear extracts 2 weeks after infection.

Local effects of $L$. major early in infection appear to delay the accumulation of leishmaniacidal IFN- $\gamma$ producers at the infection site, which could help to facilitate initial host residency by the pathogen. Interestingly, vaccine approaches that result in early accumulation of IFN- $\gamma$ producers at the lesion site result in rapid pathogen clearance (49). IFN- $\gamma$ producers can be found at the infection site later in the infection; therefore, local effects of pathogens must eventually be overridden. We propose that the early pathogen-driven exclusion of IFN- $\gamma$ producers is overridden by the magnitude of the developing acquired immune response later in infection. Between 2 and 6 weeks of infection, the total number of $L$. major-specific IFN- $\gamma$-producing cells in the dLN of a resistant B10.D2 mouse increases 20- to 40-fold. This dramatic increase in IFN- $\gamma$ effectors in the $\mathrm{LN}$ may enable a few IFN- $\gamma$-producing $\mathrm{T}$ cells to enter the infection site, establishing a positive feedback loop within the infected tissue to drive the active recruitment of Th1 immune effectors. Consistent with this model, Th1 attracting chemokine expression was detectable in the L. major-infected ear tissue at later stages of infection (Figure 1B). It is tempting to speculate that once the antigen load declines, the parasite's hold on the local microenvironment may regain dominance and facilitate long-term parasite persistence in resistant mouse strains (23).

The concept that pathogens resident in the tissue may subvert the centrally generated cytokine repertoire by limiting the local recruitment or activation of specific effectors has important implications for assessment of immune function in disease and vaccine settings. Measurement of the LN or circulating pool of antigen-specific effectors may not accurately reflect the immune 
response at the infection site, which highlights the need for better immune analysis at pathogen sites. In addition, the data suggest that, in cases where the immune response fails to control infection, the host may nevertheless have generated a central pool of appropriate effectors with potential for pathogen clearance. Novel therapies that focus on manipulating the local infection site to encourage appropriate recruitment or activation of these effectors may be particularly beneficial.

\section{Methods}

Mice. Mice were purchased through the National Cancer Institute, NIH, or from the Jackson Laboratory. BALB/c LACK-specific TCR Tg ${ }^{+}$mice (ABLE-R) were also TCR $\mathrm{C}^{-1}$. BALB/c IL-4 reporter mice (4get) were kindly provided by Richard Locksley (UCSF, San Francisco, California, USA). Mice were maintained in the pathogen-free animal care facility of the University of Rochester Medical Center.

Parasites and antigens. Metacyclic L. major promastigotes (strain WHOM/ IR/-/173) were isolated from stationary phase cultures by negative selection using peanut agglutinin (Sigma-Aldrich) (62). SLA were prepared by freeze-thaw as described previously (62).

Infections and immunizations. Cohorts of 4-10 mice were infected intradermally in the ear with $2 \times 10^{5}$ parasites in $10 \mu \mathrm{l}$ PBS. For third party experiments, mice were infected in one ear with $L$. major and immunized in the contralateral ear with $10 \mu \mathrm{g}$ OVA protein emulsified in CFA or IFA. Two weeks after infection, the mice were challenged in the L. majorinfected ear with $10 \mu \mathrm{g}$ OVA protein in PBS or with a mixture of $90 \mu \mathrm{g}$ CpG 1826 oligonucleotide (5'-TCATGACGTTCCTGACGTT) (Coley Pharmaceuticals) and $10 \mu \mathrm{g}$ OVA protein. Mice were sacrificed 30 hours after challenge for cytokine analysis. SLA immunizations used $2 \times 10^{5}$ parasite equivalents in CFA or IFA per dermal injection. The polyclonal anti-CCL7 Ab (41) or control polyclonal rabbit Ig (Sigma-Aldrich) was administered directly into the ear dermis $(250 \mu \mathrm{g} /$ ear $)$. Ear tissue was harvested 3 days after $\mathrm{Ab}$ treatment.

In vitro macrophage culture. Peritoneal macrophage cultures were isolated 5-6 days after 3\% thioglycollate injection. The peritoneum was washed, and macrophages were cultured overnight. Macrophages (75\%-94\% CD3$\mathrm{CDllb}^{+} \mathrm{F} 4 / 80^{+}$) were infected with $L$. major promastigotes (10 parasites/ macrophage). Five hours after infection, $3 \mu \mathrm{g}$ CpG 1826 was added to the cultures; 24 hours after infection, supernatants were removed and mRNA extracted from macrophages for real-time RT-PCR analysis.

Cell purification. Single cell suspensions were isolated from retromaxillary LNs and ear tissue. Ears were split into dorsal and ventral sides and incubated in $1 \mathrm{mg} / \mathrm{ml}$ dispase/collagenase (Roche) for 30 minutes at $37^{\circ} \mathrm{C}$. Cells were cultured in complete RPMI 1640 with $10 \%$ heat-inactivated FCS. T cells were depleted from single cell suspensions of APCs via positive selection using magnetically assisted chemical separation for Thy $1.2^{+}$cells.

1. Marzo, A.L., Vezys, V., Williams, K., Tough, D.F., and Lefrancois, L. 2002. Tissue-level regulation of Th1 and Th2 primary and memory CD $4 \mathrm{~T}$ cells in response to Listeria infection. J. Immunol. 168:4504-4510.

2. Debes, G.F., etal.2006. Chemotactic responses of IL-4-, IL-10-, and IFN-gamma-producing CD4+ T cells depend on tissue origin and microbial stimulus. J. Immunol. 176:557-566.

3. Wohlleben, G., et al. 2003. Influenza A virus infection inhibits the efficient recruitment of Th2 cells into the airways and the development of airway eosinophilia. J. Immunol. 170:4601-4611.

4. Matzinger, P. 2007. Friendly and dangerous signals: is the tissue in control? Nat. Immunol. 8:11-13.

5. Raz, E. 2007. Organ-specific regulation of innate
In vitro cell culture and cytokine analysis. Single-cell suspensions from $L N$ and ear tissue were cultured on anti-cytokine $\mathrm{mAb}$-coated plates in the presence or absence of SLA (equivalent to $2 \times 10^{6}$ organisms $/ \mathrm{ml}$ ) or $5 \mu \mathrm{M}$ OVA peptide 323-339 for 6 hours for IL- 4 and IFN- $\gamma$ measurement by ELISPOT as described previously (62). Control uninfected ears processed in the same way routinely yielded $<10$ cytokine-producing spots per ear.

For ex vivo cytokine analysis, the Cytokine Capture Assay (Miltenyi) was performed essentially as described previously (62). Single cell suspensions were isolated directly ex vivo from the ear 2 weeks after infection with L. major. Cells were labeled with the bifunctional Ab "catch" reagent for 5 min on ice and warmed to $37^{\circ} \mathrm{C}$ with SLA for 45 minutes for cytokine secretion. Cytokine was detected by FACS using a second anti-cytokine $\mathrm{mAb}$. Gates were drawn on cells labeled without the catch reagent ("no catch"). Th1 and Th2 effectors were generated from DO11.10 TCR Tg ${ }^{+}$ CD4 $\mathrm{T}$ cells in vitro as described previously (62).

For cytokine analysis after in vitro culture, $\mathrm{LN}$ and ear cell suspensions $\left(10^{7} / \mathrm{ml}\right)$ were incubated with $5 \mu \mathrm{M}$ CFSE (Molecular Probes) for $5 \mathrm{~min}$ at room temperature and washed 3 times before culture. Cells were incubated for 72 hours at $37^{\circ} \mathrm{C}$ with or without exogenous SLA. Intracellular cytokine staining was performed using a BD Pharmingen kit. Brefeldin A was added to the cultures 4 hours before harvest.

Chemokine analysis. Ears were surgically removed, placed on ice, and homogenized in TRIzOL (Invitrogen) for extraction of mRNA and then reverse-transcribed (RT for PCR kit; BD Clontech). Real-time RT-PCR used Assays-on-Demand Taqman primer/probe sets with an ABI prism 7900 sequence detection system (Applied Biosystems). Target levels were normalized to HPRT.

Statistics. All statistical analyses used the Mann-Whitney $U$ test unless otherwise stated. A $P$ value of less than or equal to 0.05 was considered significant. Where the Student's $t$ test was used, all analyses were 2 tailed.

\section{Acknowledgments}

We thank Stephen W. Chensue (Department of Pathology, University of Michigan, Ann Arbor, Michigan, USA) for the kind gift of antiCCL7 Ab, the Fowell laboratory for helpful suggestions, and Andrea Sant, Nick Crispe, and Jim Miller for insightful comments on the manuscript. This work was supported by NIH grants R01-AI50201 (to D.J. Fowell) and T32-A107285 (to S.D. Katzman).

Received for publication July 3, 2007, and accepted in revised form November 14, 2007.

Address correspondence to: Deborah J. Fowell, David H. Smith Center for Vaccine Biology and Immunology, University of Rochester, 601 Elmwood Avenue, Box 609, Rochester, New York 14642, USA. Phone: (585) 273-3680; Fax: (585) 273-2452; E-mail: deborah_fowell@urmc.rochester.edu. immunity. Nat. Immunol. 8:3-4.

6. Steinman, R.M., and Hemmi, H. 2006. Dendritic cells: translating innate to adaptive immunity. Curr. Top. Microbiol. Immunol. 311:17-58.

7. Kabelitz, D., and Medzhitov, R. 2007. Innate immunity-cross-talk with adaptive immunity through pattern recognition receptors and cytokines. Curr. Opin. Immunol. 19:1-3.

8. Sallusto, F., and Lanzavecchia, A. 2000. Understanding dendritic cell and T-lymphocyte traffic through the analysis of chemokine receptor expression. Immunol. Rev. 177:134-140.

9. Mora, J.R., et al. 2003. Selective imprinting of guthoming T cells by Peyer's patch dendritic cells. Nature. 424:88-93.

10. Campbell, D.J., and Butcher, E.C. 2002. Rapid acquisition of tissue-specific homing phenotypes by CD4(+) T cells activated in cutaneous or mucosal lymphoid tissues. J. Exp. Med. 195:135-141.

11. Mathew, A., et al. 2001. Signal transducer and activator of transcription 6 controls chemokine production and $\mathrm{T}$ helper cell type 2 cell trafficking in allergic pulmonary inflammation. J. Exp. Med. 193:1087-1096.

12. Mikhak, Z., et al. 2006. STAT1 in peripheral tissue differentially regulates homing of antigen-specific Th1 and Th2 cells. J. Immunol. 176:4959-4967.

13. Masopust, D., Vezys, V., Marzo, A.L., and Lefrancois, L. 2001. Preferential localization of effector memory cells in nonlymphoid tissue. Science. 291:2413-2417.

14. Harris, N.L., Watt, V., Ronchese, F., and Le Gros, G. 
2002. Differential $\mathrm{T}$ cell function and fate in lymph node and nonlymphoid tissues. J. Exp. Med. 195:317-326.

15. Mohrs, K., Wakil, A.E., Killeen, N., Locksley, R.M., and Mohrs, M. 2005. A two-step process for cytokine production revealed by IL-4 dual-reporter mice. Immunity. 23:419-429.

16. Takabayshi, K., et al. 2006. Induction of a homeostatic circuit in lung tissue by microbial compounds. Immunity. 24:475-487.

17. Johansson, C., and Kelsall, B.L. 2005. Phenotype and function of intestinal dendritic cells. Semin. Immunol. 17:284-294.

18. Laouar, A., et al. 2005. CD70+ antigen-presenting cells control the proliferation and differentiation of $\mathrm{T}$ cells in the intestinal mucosa. Nat. Immunol. 6:698-706.

19. Erb, K.J., Holloway, J.W., Sobeck, A., Moll, H., and Le Gros, G. 1998. Infection of mice with Mycobacterium bovis-Bacillus Calmette-Guerin (BCG) suppresses allergen-induced airway eosinophilia. J. Exp. Med. 187:561-569.

20. Rakasz, E., et al. 1998. Localization and regulation of IFN-gamma production within the granulomas of murine schistosomiasis in IL-4-deficient and control mice. J. Immunol. 160:4994-4999.

21. Mantovani, A., Bonecchi, R., and Locati, M. 2006. Tuning inflammation and immunity by chemokine sequestration: decoys and more. Nat. Rev. Immunol. 6:907-918.

22. Smith, P., et al. 2005. Schistosoma mansoni secretes a chemokine binding protein with antiinflammatory activity. J. Exp. Med. 202:1319-1325.

23. Sacks, D., and Noben-Trauth, N. 2002. The immunology of susceptibility and resistance to Leishmania major in mice. Nat. Rev. Immunol. 2:845-858.

24. Reiner, S.L., Zheng, S., Wang, Z.E., Stowring, L., and Locksley, R.M. 1994. Leishmania promastigotes evade interleukin 12 (IL-12) induction by macrophages and stimulate a broad range of cytokines from CD4+ T cells during initiation of infection. J. Exp. Med. 179:447-456.

25. Carrera, L., et al. 1996. Leishmania promastigotes selectively inhibit interleukin 12 induction in bone marrow-derived macrophages from susceptible and resistant mice. J. Exp. Med. 183:515-526.

26. Olivier, M., Gregory, D.J., and Forget, G. 2005. Subversion mechanisms by which Leishmania parasites can escape the host immune response: a signaling point of view. Clin. Microbiol. Rev. 18:293-305.

27. Cameron, P., et al. 2004. Inhibition of lipopolysaccharide-induced macrophage IL-12 production by Leishmania mexicana amastigotes: the role of cysteine peptidases and the NF-kappaB signaling pathway. J. Immunol. 173:3297-3304.

28. Belkaid, Y., Mendez, S., Lira, R., Kadambi, N., Milon, G., and Sacks, D. 2000. A natural model of Leishmania major infection reveals a prolonged "silent" phase of parasite amplification in the skin before the onset of lesion formation and immunity. J. Immunol. 165:969-977.

29. Belkaid, Y., Butcher, B., and Sacks, D.L. 1998. Analysis of cytokine production by inflammatory mouse macrophages at the single-cell level: selective impairment of IL-12 induction in Leishmaniainfected cells. Eur J. Immunol. 28:1389-1400.

30. Menten, P., Wuyts, A., and Van Damme, J. 2001. Monocyte chemotactic protein-3. Eur. Cytokine Netw. 12:554-560.

31. Kawai, T., et al. 1999. Selective diapedesis of Th1 cells induced by endothelial cell RANTES. J. Immunol. 163:3269-3278.

32. Belkaid, Y., Piccirillo, C.A., Mendez, S., Shevach, E.M., and Sacks, D.L. 2002. CD4+CD25+ regulatory $\mathrm{T}$ cells control Leishmania major persistence and immunity. Nature. 420:502-507.
33. Scott, P., Eaton, A., Gause, W.C., di Zhou, X., and Hondowicz, B. 1996. Early IL-4 production does not predict susceptibility to Leishmania major. Exp. Parasitol. 84:178-187.

34. Stetson, D.B., et al. 2002. Rapid expansion and IL-4 expression by Leishmania-specific naive helper $\mathrm{T}$ cells in vivo. Immunity. 17:191-200.

35. Zaph, C., Uzonna, J., Beverley, S.M., and Scott, P. 2004. Central memory $T$ cells mediate long-term immunity to Leishmania major in the absence of persistent parasites. Nat. Med. 10:1104-1110.

36. Mohrs, M., Shinkai, K., Mohrs, K., and Locksley, R.M. 2001. Analysis of type 2 immunity in vivo with a bicistronic IL-4 reporter. Immunity. 15:303-311.

37. Nicolas, L., Sidjanski, S., Colle, J.H., and Milon, G. 2000. Leishmania major reaches distant cutaneous sites where it persists transiently while persisting durably in the primary dermal site and its draining lymph node: a study with laboratory mice. Infect. Immun. 68:6561-6566.

38. Romagnani, S. 2002. Cytokines and chemoattractants in allergic inflammation. Mol. Immunol. 38:881-885.

39. Blanpain, C., et al. 1999. CCR5 binds multiple CCchemokines: MCP-3 acts as a natural antagonist. Blood. 94:1899-1905.

40. Braun, M.C., Lahey, E., and Kelsall, B.L. 2000. Selective suppression of IL-12 production by chemoattractants. J. Immunol. 164:3009-3017.

41. Shang, X.Z., et al. 2002. Eosinophil recruitment in type-2 hypersensitivity pulmonary granulomas: source and contribution of monocyte chemotactic protein-3 (CCL7). Am. J. Pathol. 161:257-266.

42. Vannier-Santos, M.A., Martiny, A., and de Souza, W. 2002. Cell biology of Leishmania spp.: invading and evading. Curr. Pharm. Des. 8:297-318.

43. Martiny, A., Meyer-Fernandes, J.R., de Souza, W., and Vannier-Santos, M.A. 1999. Altered tyrosine phosphorylation of ERK1 MAP kinase and other macrophage molecules caused by Leishmania amastigotes. Mol. Biochem. Parasitol. 102:1-12.

44. Guizani-Tabbane, L., Ben-Aissa, K., Belghith, M., Sassi, A., and Dellagi, K. 2004. Leishmania major amastigotes induce p50/c-Rel NF-kappa $B$ transcription factor in human macrophages: involvement in cytokine synthesis. Infect. Immun. 72:2582-2589.

45. Awasthi, A., et al. 2003. CD40 signaling is impaired in L. major-infected macrophages and is rescued by a p38MAPK activator establishing a host-protective memory T cell response. J. Exp. Med. 197:1037-1043.

46. Matte, C., and Olivier, M. 2002. Leishmaniainduced cellular recruitment during the early inflammatory response: modulation of proinflammatory mediators. J. Infect. Dis. 185:673-681.

47. Ji, J., Sun, J., and Soong, L. 2003. Impaired expression of inflammatory cytokines and chemokines at early stages of infection with Leishmania amazonensis. Infect. Immun. 71:4278-4288.

48. Antoniazi, S., et al. 2004. Chemokine gene expression in toll-like receptor-competent and -deficient mice infected with Leishmania major. Infect. Immun. 72:5168-5174.

49. Mendez, S., et al. 2003. Coinjection with CpG-containing immunostimulatory oligodeoxynucleotides reduces the pathogenicity of a live vaccine against cutaneous Leishmaniasis but maintains its potency and durability. Infect. Immun. 71:5121-5129.

50. Shah, J.A., et al. 2003. Dendritic cells are responsible for the capacity of $\mathrm{CPG}$ oligodeoxynucleotides to act as an adjuvant for protective vaccine immunity against Leishmania major in mice. J. Exp. Med. 198:281-291.

51. Teixeira, M.J., Teixeira, C.R., Andrade, B.B., Barral-Netto, M., and Barral, A. 2006. Chemokines in host-parasite interactions in leishmaniasis. Trends
Parasitol. 22:32-40.

52. Serezani, C.H., et al. 2002. Evaluation of the murine immune response to Leishmania meta 1 antigen delivered as recombinant protein or DNA vaccine. Vaccine. 20:3755-3763.

53. Anderson, C.F., Oukka, M., Kuchroo, V.J., and Sacks, D. 2007. CD4(+)CD25(-)Foxp3(-) Th1 cells are the source of IL-10-mediated immune suppression in chronic cutaneous leishmaniasis. J. Exp. Med. 204:285-297.

54. Belkaid, Y., et al. 2001. The role of interleukin (IL)10 in the persistence of Leishmania major in the skin after healing and the therapeutic potential of anti-IL-10 receptor antibody for sterile cure. J. Exp. Med. 194:1497-1506.

55. Zaph, C., and Scott, P. 2003. Interleukin-12 regulates chemokine gene expression during the early immune response to Leishmania major. Infect. Immun. 71:1587-1589.

56. Park, A.Y., Hondowicz, B.D., and Scott, P. 2000. IL-12 is required to maintain a Th1 response during Leishmania major infection. J. Immunol. 165:896-902.

57. Stobie, L., et al. 2000. The role of antigen and IL-12 in sustaining Th1 memory cells in vivo: IL-12 is required to maintain memory/effector Th1 cells sufficient to mediate protection to an infectious parasite challenge. Proc. Natl. Acad. Sci. U. S. A. 97:8427-8432.

58. Yap, G., Pesin, M., and Sher, A. 2000. Cutting edge: IL-12 is required for the maintenance of IFNgamma production in $\mathrm{T}$ cells mediating chronic resistance to the intracellular pathogen, Toxoplasma gondii. J. Immunol. 165:628-631.

59. Feng, C.G., et al. 2005. Maintenance of pulmonary Th1 effector function in chronic tuberculosis requires persistent IL-12 production. J. Immunol. 174:4185-4192.

60. Wurtz, O., Bajenoff, M., and Guerder, S. 2004. IL-4mediated inhibition of IFN-gamma production by CD4+ T cells proceeds by several developmentally regulated mechanisms. Int. Immunol. 16:501-508.

61. Friedman, R.S., Jacobelli, J., and Krummel, M.F. 2006. Surface-bound chemokines capture and prime T cells for synapse formation. Nat. Immunol. 7:1101-1108.

62. Au-Yeung, B.B., Katzman, S.D., and Fowell, D.J. 2006. Cutting edge: Itk-dependent signals required for CD4+ T cells to exert, but not gain, Th2 effector function. J. Immunol. 176:3895-3899.

63. Yamashita, M., et al. 2006. Crucial role of MLL for the maintenance of memory $\mathrm{T}$ helper type 2 cell responses. Immunity. 24:611-622.

64. Tykocinski, L.O., et al. 2005. A critical control element for interleukin-4 memory expression in $\mathrm{T}$ helper lymphocytes. J. Biol. Chem. 280:28177-28185.

65. Guo, L., Hu-Li, J., and Paul, W.E. 2004. Probabilistic regulation of IL-4 production in Th2 cells: accessibility at the Il4 locus. Immunity. 20:193-203.

66. Morales-Tirado, V., et al. 2004. Cutting edge: selective requirement for the Wiskott-Aldrich syndrome protein in cytokine, but not chemokine, secretion by CD4+ T cells. J. Immunol. 173:726-730.

67. Bezbradica, J.S., et al. 2006. Granulocyte-macrophage colony-stimulating factor regulates effector differentiation of invariant natural killer T cells during thymic ontogeny. Immunity. 25:487-497.

68. Reinhardt, R.L., Bullard, D.C., Weaver, C.T., and Jenkins, M.K. 2003. Preferential accumulation of antigen-specific effector CD4 T cells at an antigen injection site involves CD62E-dependent migration but not local proliferation. J. Exp. Med. 197:751-762.

69. Dustin, M.L., Bromley, S.K., Kan, Z., Peterson, D.A., and Unanue, E.R. 1997. Antigen receptor engagement delivers a stop signal to migrating $\mathrm{T}$ lymphocytes. Proc. Natl. Acad. Sci. U. S. A. 94:3909-3913. 